\title{
VISUAL FUNCTION IN WEST SYNDROME
}

The range and severity of visual abnormalities in 25 infants with West syndrome, and their relation to seizures, EEG patterns and brain lesions were studied at Catholic University, Rome; University of Pisa; and University of Pavia, Italy. All had normal funduscopic examinations. Tests of visual function (acuity, visual field, fixation, pursuit, eye movement, and visual attention) showed at least one abnormal result on one or more of the tests in 22 children. At spasm onset, visual impairment was related to EEG sleep disorganization rather than the hypsarrhythmic pattern. After 2 months, both EEG features were significantly correlated with visual function. With seizure control after 2 months, visual function improved in several cases. Cryptogenic and tuberous sclerosis patients had good visual function, whereas other patients with symptomatic spasms had more frequent visual dysfunction. The most severe abnormalities of visual function occurred with lesions involving the basal ganglia, posterior cortical areas, and optic radiations, but some infants with normal MRIs also had impaired visual function. Fluctuations in visual performance of fixation and pursuit in 17 patients were not related to the early EEG changes. Hypsarrhytmia was significantly related to low scores in visual function only after the first 2 months. Improvement in visual function after 2 months was correlated with good or partial control of seizures in 9 cases and only 4 without seizure control. (Rando T, Bancale A, Baranello, et al. Visual function in infants with West syndrome: correlation with EEG patterns. Epilepsia 2004;45:781-786). (Reprints: Dr F Guzzetta, Division of Child Neurology and Psychiatry, Catholic University, Rome, Italy).

COMMENT. Visual dysfunction in infants with West syndrome is caused by various factors and not only by brain structural abnormalities. The epileptic disorder per se is correlated with visual function. In drug or ACTH-responsive cases (idiopathic infantile spasms) a transitory hypsarrhythmia is predictive of normal visual function, whereas a persistent hypsarrhythmia and resistant spasms are risk factors for impaired visual function and attention.

Visual inatention, an early manifestation of West syndrome (WS). Jambaque et al found that visual inattention in WS was frequently associated with parieto-ocipital abnormalities in SPECT studies (Epilepsia July/Aug 1993; and Ped Neur Briefs Sept 1993); and Iinuma et al reported that two thirds of 17 infants with visual abnormalities and occipital EEG discharges developed WS with hypsarrhythmia at follow-up (Epilepsia 1994;35:806-809; Ped Neur Briefs Nov 1994).

\section{PROLONGED UNCONSCIOUSNESS AND DELIRIUM WITH FEBRILE SEIZURES}

The incidence and duration of unconsciousness and delirious behavior in children with febrile seizures were studied at Nagoya University Graduate School of Medicine and other centers in Japan. In 203 patients with 213 consecutive febrile seizures the duration of seizures was less than 5 minutes in $90.2 \%$, duration of unconsciousness was less than $30 \mathrm{~min}$ in $93 \%$, and delirious behavior observed in $2 \%$ of patients persisted for 10 to 60 minutes. Delirium appeared before the onset of the febrile seizure and resolved after the seizure. 
Prolonged unconsciousness $>30 \mathrm{~min}$, in $7 \%$ of patients, was associated with nongeneralized seizures, seizures $>5$ min duration, or use of intravenous diazepam. (Okumura A, Uemura N, Suzuki M, et al. Unconsciousness and delirious behavior in children with febrile seizures. Pediatr Neurol 2004;30:316-319). (Respond: Dr Okumura, Department of Pediatrics, Nagoya University Graduate School of Medicine, 65 Tsurumai-cho, Showa-ku, Nagoya, Aichi 466-8550, Japan).

COMMENT. Prolonged unconsciousness and delirious behavior are rare with febrile seizures. When these symptoms are present, a diagnosis of acute encephalopathy should be considered.

Delirium with febrile myoclonus. Febrile myoclonic episodes in 11 patients, aged 8 months to 11 years, were associated with fear, surprise and shouting in $73 \%$, a past history of febrile convulsion in $18 \%$ and febrile delirium in $9 \%$. The outcome for febrile myoclonus was benign and not complicated by afebrile seizures.

\section{MOVEMENT DISORDERS}

\section{POST-STREPTOCOCCAL DYSKINESIAS}

Forty children with dyskinetic movement disorders and associated psychiatric disorders following streptococcal infections seen between 1999 and 2002 are reported from the Institute of Child Health and Great Ormond Street Hospital for Children, London, UK. Dyskinesias included chorea in 20 patients (65\% female), motor tics (16 [69\% male]), dystonia (5), tremor (3), stereotypies (2), opsoclonus (2), and myoclonus (1). Psychiatric disorders were diagnosed in $62.5 \%$, compared to an $8.9 \%$ expected incidence in UK children. These included emotional disorders in $47.5 \%$, including obsessive-compulsive disorder $(27.5 \%)$, anxiety $(25 \%)$, and depressive episodes in $17.5 \%$. Conduct disorder occurred in $27.5 \%$ and hyperkinetic behavior in $15 \%$. A family history of dyskinesias, psychiatric disorders, and post-streptococcal autoimmune disorders was common. Symptoms continued in $72.5 \%$ at a mean follow up of 2.7 years. (Dale RC, Heyman I, Surtees RAH, et al. Dyskinesias and associated psychiatric disorders following streptococcal infections. Arch Dis Child 2004;89:604-610). (Respond: Dr R Dale, Neurosciences Unit, Wolfson Centre, Institute of Child Health, Mecklenburgh Square, London WC1N 2AP, UK).

COMMENT. The authors have extended their earlier report of a postinfectious autoimmunity against basal ganglia associated with an encephalitis lethargica syndrome (Dale et al. Brain 2004;127:21-33; Ped Neur Briefs Jan 2004;18:1-2) to emphasize the association of dyskinesias and psychiatric disorders following streptococcal infection in children. In a further article, they have demonstrated the utility of anti-basal ganglia (neuronal) antibodies (ABGA), using Western immunoblotting, as a potential diagnostic marker in post-streptococcal neurological disorders (Church AJ, Dale RC, Giovannoni G. Arch Dis Child 2004;89:611-614). That an autoimmune mechanism may underlie a proportion of adult cases of atypical movement disorders is suggested by the finding of ABGA in $42(65 \%)$ of 65 consecutive patients seen at the Institute of Neurology, Queen Square, London (Edwards MJ et al. Neurology July (1 of 2) 2004;63:156-158). Multiple factors may be involved in the mechanism of post-streptococcal dyskinesias. 